
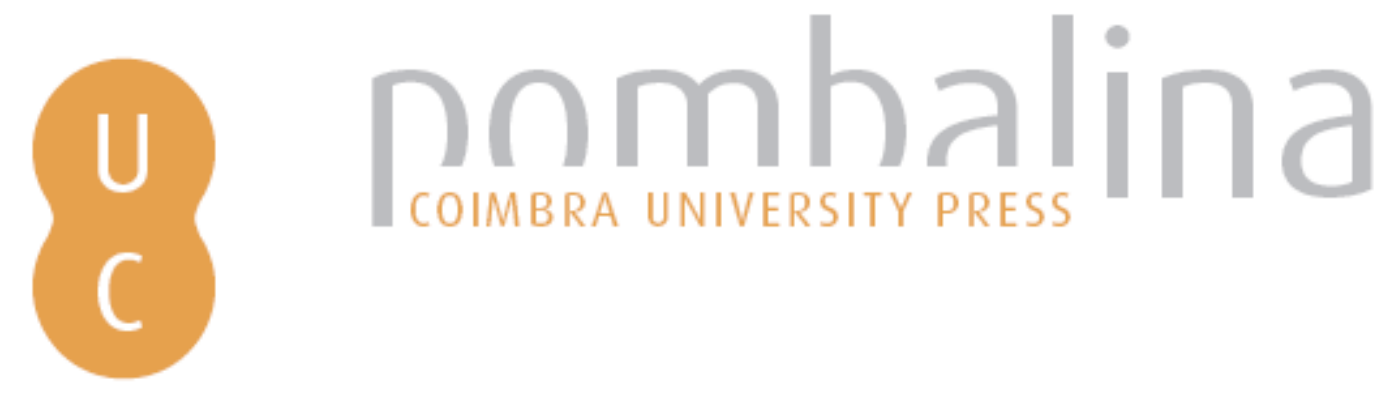

\title{
Percepções de estudantes universitários face às doenças mentais pré e pós Movimento Upa 08
}
Autor(es):
Almeida, Marina Melo; Campos, Luísa; Palha, Filipa
Publicado por: Imprensa da Universidade de Coimbra
URL
persistente:
URI:http://hdl.handle.net/10316.2/35963
DOI:
DOI:http://dx.doi.org/10.14195/978-989-26-0852-5_10
Accessed : $\quad$ 26-Apr-2023 12:23:03

A navegação consulta e descarregamento dos títulos inseridos nas Bibliotecas Digitais UC Digitalis, UC Pombalina e UC Impactum, pressupõem a aceitação plena e sem reservas dos Termos e Condições de Uso destas Bibliotecas Digitais, disponíveis em https://digitalis.uc.pt/pt-pt/termos.

Conforme exposto nos referidos Termos e Condições de Uso, o descarregamento de títulos de acesso restrito requer uma licença válida de autorização devendo o utilizador aceder ao(s) documento(s) a partir de um endereço de IP da instituição detentora da supramencionada licença.

Ao utilizador é apenas permitido o descarregamento para uso pessoal, pelo que o emprego do(s) título(s) descarregado(s) para outro fim, designadamente comercial, carece de autorização do respetivo autor ou editor da obra.

Na medida em que todas as obras da UC Digitalis se encontram protegidas pelo Código do Direito de Autor e Direitos Conexos e demais legislação aplicável, toda a cópia, parcial ou total, deste documento, nos casos em que é legalmente admitida, deverá conter ou fazer-se acompanhar por este aviso. 

Marina Melo Almeida ${ }^{1}$, Luísa Campos² \& Filipa Palha ${ }^{3}$

\section{PERCEPÇÕ ES DE ESTUDANTES UNIVERSITÁRIOS FACE ÀS DOENÇAS MENTAIS PRÉ E PÓS MOVIMENTOUPA O 8}

\section{PARTE I}

\section{Enquadramento do Projecto}

As pessoas com doença mental deparam-se frequentemente com um duplo problema. Por um lado, têm de lidar com os sintomas e as dificuldades que a própria doença lhes causa, por outro lado, são alvo de estigma, que resulta de interpretações sociais incorrectas (Corrigan \& Watson, 2002; Rüsch, Angermeyer \& Corrigan, 2005) e conduz a uma desvalorização dos indivíduos com este tipo de doença.

As atitudes e comportamentos negativos face às pessoas com doença mental estiveram presentes ao longo dos tempos (Stuart, 2003). Este tipo de atitudes foi identificado como um obstáculo para a reabilitação da doença e para a integração plena da pessoa na sociedade (Thornicoft, 2006 cit. in Markström et al., 2009).

No sentido de reduzir o estigma associado à doença mental têm sido desenvolvidas, nos últimos anos, algumas campanhas e abordagens de combate ao estigma, que incluem a promoção da educação/formação da

\footnotetext{
${ }^{1}$ Universidade Católica Portuguesa. E-mail: marina_i_m_almeida@hotmail.com

2 Professora Auxiliar da Faculdade de Educação e Psicologia da Universidade Católica. Membro da Direcção da ENCONTRAR+SE.

3 Docente da Faculdade de Educação e Psicologia da Universidade Católica. Presidente da Direcção da ENCONTRAR+SE - Associação de apoio às pessoas com perturbação mental grave.
} 
população para esta problemática, o protesto contra a discriminação e o contacto interpessoal com indivíduos com este tipo de doença (Corrigan \& Penn, 1999; Corrigan \& Watson, 2002). Algumas abordagens foram criadas especialmente para grupos específicos dentro da população geral: adolescentes, licenciados, estudantes universitários, entre outros.

Este estudo insere-se no contexto de uma campanha anti-estigma/pró saúde mental pioneira em Portugal e tem como objectivo geral avaliar as percepções dos estudantes universitários face à doença mental para compreender possíveis necessidades de intervenções educativas.

\section{O estudo do estigma público}

Este estudo centra-se no estigma público pelo que se considera importante fazer referência àquilo que tem sido levado a cabo para avaliar este tipo de estigma, no sentido de posteriormente se poder intervir. Para isso, destacam-se alguns estudos e algumas sondagens que tiveram como objectivo o levantamento de dados acerca das atitudes das populações face às pessoas com doença mental. É o caso da sondagem realizada no Reino Unido, em 1998, antes do início da campanha anti-estigma promovida pelo Royal College of Psychiatrists. Com esta sondagem procurou-se, assim, avaliar as atitudes da opinião pública deste país relativamente aos indivíduos com doença mental. Isto porque até à data, muitos estudos tinham já demonstrado que as atitudes estigmatizantes face às pessoas com doença mental eram comuns, porém não existiam estudos recentes que incluíssem uma amostra grande e representativa da população inglesa (Crisp, Gelder, Rix, Meltzer \& Rowlands, 2000). Este estudo incluiu uma amostra de 1737 adultos que reponderam a entrevistas. Nestas perguntava-se acerca de 8 tópicos que eram repetidos para 7 doenças mentais: depressão grave, ataques de pânico e fobias, demência, perturbação do comportamento alimentar, esquizofrenia, alcoolismo e toxicodependência. Os resultados desta sondagem revelam que a esquizofrenia, o alcoolismo e a toxicodependência são as doenças alvo de atitudes mais negativas. (Crisp, Gelder, Rix, Meltzer \& Rowlands, 2000). 
Destaca-se também uma sondagem que é parte integrante de um estudo piloto realizado em 1996, no Canadá, com o objectivo de avaliar as atitudes da população face às pessoas com esquizofrenia e que permitiu fazer o levantamento de necessidades que possibilitasse o planeamento de uma campanha anti-estigma promovida pela World Psychiatric Association em diversos países (Thompson, Stuart, Bland, Arboleda-Florez, Warner \& Dickson, 2002). Os resultados deste estudo piloto mostraram que o público em geral estava bem informado sobre a esquizofrenia (Stuart \& Arboleda-Flórez, 2001; Thompson, Stuart, Bland, Arboleda-Florez, Warner \& Dickson, 2002). A maioria das pessoas conseguiu identificar uma causa biológica desta doença e apoiava o tratamento na comunidade (Stuart \& Arboleda-Flórez, 2001). Contudo, ter maior conhecimento não é sinónimo de ter atitudes de maior aceitação, uma vez que, tendo em conta o estudo de Stuart e Arboleda-Flórez (2001), as pessoas que trabalhavam numa instituição de saúde mental, ainda que tivessem maior informação sobre esta temática, revelaram atitudes tão estigmatizantes como qualquer outro grupo de profissionais.

\subsection{Abordagens de combate ao estigma público}

Combater o estigma e a discriminação é sinónimo de lutar pela saúde mental (Stuart, 2005). O estigma foi considerado pela World Health Organization (2001) como a única e mais importante barreira a ultrapassar na comunidade. Para combater o estigma e a discriminação são necessárias múltiplas abordagens que envolvem a educação/formação dos profissionais de saúde, o encerramento de instituições psiquiátricas que servem apenas para manter a segregação e reforçar o estigma, a existência de serviços de saúde mental na comunidade e a implementação de legislação para proteger os direitos dos indivíduos com doença mental (World Health Organization, 2001).

Assim, desenvolveram-se algumas estratégias de diminuição do estigma público. Estas estratégias agrupam-se em três abordagens: protesto, educação e contacto (Corrigan \& Penn, 1999; Corrigan \& Watson, 2002). Os 
esforços de protesto desafiam estereótipos sociais, reivindicando contra os meios de comunicação que difundem representações sociais incorrectas face à doença mental e contra as opiniões negativas das populações em relação a este tipo de doença (Corrigan \& Watson, 2002). A educação pode fornecer informação para que se tomem decisões mais esclarecidas acerca da doença mental. A literatura indica que é menos provável que as pessoas que demonstram um maior conhecimento sobre a doença mental defendam comportamentos discriminatórios (Roman, Floyd, Link \& Cullen, 1981). Com efeito, os programas educativos são eficazes para diversos grupos, incluindo estudantes universitários, licenciados, adolescentes e residentes comunitários. Por último, a estratégia do contacto: o facto de se ter contacto directo com alguém com doença mental pode reduzir o estigma, especialmente quando este tipo de contacto é estabelecido com um indivíduo que esteja integrado na comunidade (Corrigan \& Watson, 2002). A luta contra o estigma requer também campanhas de informação pública que transmitam conhecimento às comunidades acerca da natureza, da dimensão e do impacto da doença mental, no intuito de se afastarem alguns mitos e de se fomentar o desenvolvimento de atitudes e comportamentos mais positivos (World Health Organization, 2001).

É com estes propósitos que nascem algumas campanhas anti-estigma criadas por organizações internacionais, como a World Psychiatric Association (Thompson, Stuart, Bland, Arboleda-Florez, Warner \& Dickson, 2002), ou por associações nacionais, tais como o Royal College of Psychiatrists no Reino Unido (Crisp, Gelder, Rix, Meltzer \& Rowlands, 2000) e o National Mental Health Promotion and Prevention Action Plan (Rosen, Walter, Casey \& Hocking, 2000 cit. in Stuart, 2003).

A World Psychiatric Association lançou uma campanha global no $21 .^{\circ}$ congresso mundial de psiquiatria na Alemanha, para combater o estigma e a discriminação face à esquizofrenia. Previamente a esta campanha foi realizado um estudo piloto no Canadá ao qual foi feito referência anteriormente. O programa piloto implementou uma variedade de intervenções dirigidas aos meios de comunicação social, às escolas e ao público em geral, avaliou os seus resultados e desenvolveu orientações baseadas nos resultados obtidos (Stuart, 2003). Posteriormente, a campanha desenvolvida 
pela World Psychiatric Association estendeu-se a 27 países ao abrigo do programa Open the doors, países que foram estabelecendo iniciativas de redução do estigma (Thompson, Stuart, Bland, Arboleda-Florez, Warner \& Dickson, 2002; Stuart, 2003).

Uma outra campanha à qual pode ser feita referência é a campanha Changing Minds, organizada pelo Royal College of Psychiatrists que teve início em 1998, tendo tido uma duração de 5 anos. Os seus objectivos prendem-se com o incremento do conhecimento público e profissional acerca da doença mental e com a redução do estigma e discriminação associados. O programa tem dois principais temas: o primeiro refere-se à necessidade de mudar mentalidades, para mudar atitudes; e o segundo enfatiza o elevado burden associado à doença mental e como este, directa ou indirectamente, acaba por afectar as nossas vidas. Este programa desenvolveu alguns materiais criados para encorajar as pessoas a parar, a pensar e a compreender a doença mental. Isto incluiu a distribuição de panfletos para a população em geral, a distribuição de pequenos livros para professores e alunos, a criação de websites, posters e vídeos (Stuart, 2003). Antes desta campanha começar, foi também realizada uma sondagem, em 1998, anteriormente referida, para avaliar as atitudes predominantes da população face a 7 doenças mentais. No final da campanha, em 2003, foi novamente realizada uma sondagem para avaliar os efeitos desta mesma.

Em relação ao National Mental Health Promotion and Prevention Action Plan implementado na Austrália, em 1999, este teve como objectivo o aumento do grau de instrução da população sobre doença mental. O programa teve a duração de 4 anos e desenvolveu uma campanha pública nacional. Também se fizeram sondagens antes e depois da campanha para se avaliar atitudes e monitorizar resultados (Rosen, Walter, Casey \& Hocking, 2000, cit. in Stuart, 2003).

Segundo Stuart (2003), o sucesso deste tipo de campanhas vai depender da aceitação da comunidade à sua implementação. Isto porque as reacções comunitárias têm sido dos factores mais importantes do período da desinstitucionalização. As reacções negativas, não só limitam a possibilidade dos indivíduos com doença mental participarem na vida comunitária, como 
também limitam o estabelecimento e a eficácia deste tipo de programas comunitários pró saúde mental (Rabkin, Muhlin, \& Cohn, 1984; Segal, Baumohl \& Moyles, 1980). A aceitação pública do tratamento da doença mental na comunidade e a rejeição social das pessoas com este tipo de doença estão muitas vezes relacionadas com percepções de perigosidade e de imprevisibilidade (Trute, Teft \& Segall, 1989 cit. in Stuart 2003).

\subsection{Estudos com estudantes universitários}

Alguns dos estudos sobre estigma público e, como já referido, algumas das abordagens de combate ao estigma estão direccionados especificamente para diferentes grupos dentro da população em geral: para adolescentes, para licenciados, para residentes comunitários, para estudantes universitários, entre outros. Estes últimos constituem a população-alvo deste estudo e pretende-se estudar as suas percepções face à doença mental. O estudo das percepções reveste-se de importância, visto que estas estão correlacionadas com as atitudes, na medida em que as atitudes desenvolvem-se com base no posicionamento do indivíduo face a estímulos que percepciona (Lima, 2002). Por sua vez, as atitudes são importantes factores de previsão do comportamento humano (Ajzen \& Fishbein, 1977; Lima, 2002).

Torna-se igualmente importante compreender as percepções dos estudantes universitários face à doença mental, uma vez que este grupo poderá vir a trabalhar, directa ou indirectamente, com uma população com este tipo de problemática, sendo fundamental o desenvolvimento de percepções e atitudes positivas que, consequentemente possam conduzir a comportamentos também favoráveis face às pessoas com doença mental. Como é defendido por Feldmann (2005), as percepções negativas dos estudantes dificultam a função de psiquiatras, de ensinar futuros médicos a reconhecer e a tratar este tipo de doenças.

Para além disto, até os profissionais de saúde demonstram estigma associado à doença mental (Sadow \& Ryder, 2008). Já em 1962, Cohen e Struening interessaram-se pelo estudo das atitudes estigmatizantes face 
à doença mental de trabalhadores de um hospital, incluindo psicólogos, psiquiatras, enfermeiros, médicos, dentistas e auxiliares. (Cohen \& Struening, 1965, cit. in Sadow e Ryder, 2008). Segundo Levey e Howells (1994 cit. in Wallach, 2004) existe estigma associado à doença mental em profissionais que trabalham com esta população, tais como enfermeiros e terapeutas ocupacionais. Estes autores revelam, por exemplo, que existe a crença prevalente entre os profissionais de saúde de que os indivíduos com esquizofrenia são violentos e imprevisíveis e que, por isso, são considerados perigosos.

Constatou-se, igualmente, que também os psicólogos desenvolvem atitudes e comportamentos negativos em relação às pessoas com doença mental. Embora existam estudos que avaliam as atitudes dos psicólogos como sendo mais positivas e mais optimistas em relação aos resultados do tratamento, do que as atitudes de outros profissionais de saúde (Roskin, Carsen, Rabiner \& Marell, 1988, cit. in Servais \& Saunders, 2007), também há evidência da existência de atitudes negativas reveladas por psicólogos clínicos. Kahle e White (1991, cit. in Servais \& Saunders, 2007) constataram que alguns psicólogos revelavam desconforto ao contactarem com indivíduos com problemas de abuso de substâncias. Para além disso, alguns deles também se mostraram relutantes em trabalhar com pessoas com doenças mentais graves, e outros tendem a distanciar-se de pessoas com perturbações de personalidade ou psicóticas (Servais \& Saunders, 2007).

O estudo de Lauber, Anthony, Ajdacic-Gross e Rossler (2004) compara atitudes de psiquiatras face à doença mental com atitudes da população em geral e concluiu que os psiquiatras revelam maior aceitação face a um aumento das facilidades ligadas à saúde mental na comunidade, porém, apresentam atitudes de distância social face às pessoas com doença mental tão elevadas como a população em geral.

No intuito de se compreender as atitudes dos estudantes universitários têm sido realizados alguns estudos. De acordo com Chung, Chen e Liu (2001), as atitudes dos estudantes de medicina relativamente às pessoas com doença mental influenciam o tratamento que estes podem fornecer aos pacientes. Estes autores desenvolveram, assim, um trabalho 
de investigação onde procuraram estudar as atitudes de 308 estudantes: 151 estudantes de medicina, 65 de engenharia, 65 de ciências sociais e 27 de medicina dentária. Os estudantes não tinham tido qualquer tipo de formação prévia acerca desta área. O instrumento utilizado foi um questionário de 19 itens (por exemplo: "tem vontade de casar com uma pessoa com doença mental?"; "tem vontade de estabelecer uma amizade com alguém com doença mental?") que avaliava a distância social face às pessoas com doença mental, usando uma escala de 4 pontos. Os resultados obtidos revelaram que os estudantes de medicina, em geral, apresentam atitudes de maior aceitação face a pessoas com doença mental quando comparados com os estudantes dos outros dois cursos (engenharia e ciências sociais); os estudantes com contacto prévio com pessoas com doença mental apresentaram atitudes mais positivas face às pessoas com doença mental (Chung, Chen \& Liu, 2001).

Pode salientar-se um outro estudo desenvolvido por Ecker (1988) cujo objectivo foi comparar as atitudes dos estudantes universitários do primeiro ano dos cursos de psicologia, de medicina e de ciências (matemática, física e química), para avaliar se os alunos de psicologia demonstravam atitudes mais favoráveis em relação às pessoas com doença mental.

A amostra era constituída por 48 estudantes de psicologia, 62 estudantes de medicina e 55 estudantes de ciências. Foi efectuada uma análise semântica diferencial para avaliar as atitudes dos estudantes. Criaram-se 28 itens que tinham como finalidade caracterizar o comportamento de uma pessoa com doença mental e que o inquirido tinha de avaliar através duma escala de 1 a 7 pontos, sendo que os extremos representavam palavras opostas, por exemplo: bom/mau; fraco/forte; responsável/irresponsável; perigoso/não perigoso; alegre/triste, entre outros. Esta análise foi utilizada, tendo por base um estudo prévio do mesmo autor.

Os resultados revelaram que as respostas dos três grupos de estudantes eram semelhantes. Dos 28 itens, só se verificaram diferenças significativas em cinco e não foram observadas diferenças significativas entre os estudantes de psicologia e os de medicina. Relativamente à comparação dos estudantes de psicologia com os de ciências apenas se verificaram diferenças significativas em três itens. 
Tendo em conta os resultados obtidos, os autores concluíram que as atitudes dos estudantes de psicologia e dos de medicina se mostravam mais semelhantes. Os resultados pareciam também indicar que um nível específico de formação era já suficiente para desenvolver atitudes semelhantes face aos indivíduos com doença mental.

Um outro estudo realizado por Javed, Naeem, Kingdon, Irfan, Izhar e Ayub (2006), procurou estudar o estigma face à doença mental entre estudantes e professores universitários. Para isso foi utilizado um questionário cujos itens se basearam na sondagem desenvolvida por Crisp Gelder, Rix, Meltzer e Rowlands (2000). Assim, foram realizadas perguntas sobre 5 doenças mentais (Esquizofrenia, Depressão, Demência, Alcoolismo e Toxicodependência) e as atitudes dos estudantes e professores universitários são avaliadas por meio de respostas a 8 itens (perigosidade, imprevisibilidade, capacidade dos médicos em falar com a pessoa, a única pessoa a culpar pela situação, sente de maneira diferente das outras pessoas, se quisesse podia melhorar a situação em que está, a pessoa pode melhorar com o tratamento, e a pessoa pode recuperar por completo).

Os resultados descritos dizem respeito somente a quatro doenças (Esquizofrenia, Depressão, Alcoolismo e Toxicodependência). Excluiu-se a Demência uma vez que apenas uma reduzida percentagem de pessoas, neste estudo, ouvira falar desta doença. Após análise dos resultados verificam-se atitudes mais negativas por parte dos estudantes universitários face à depressão no item perigosidade (77\%), face à esquizofrenia (86\% e $74,4 \%)$ e à toxicodependência $(96,6 \%$ e $86,9 \%)$ nos itens perigosidade e é a única pessoa a culpar pela situação.

Um outro estudo recente realizado na Suécia procurou avaliar a mudança de atitudes dos estudantes universitários na área da saúde (médicos, psicólogos, enfermeiros, terapeutas ocupacionais e fisioterapeutas) antes e depois destes terem tido formação teórico-prática sobre saúde mental (Markström et al., 2009). Nesta investigação foi usada uma versão sueca do questionário "Attitudes to Persons with Mental Illness", utilizado no âmbito da campanha Changing Minds que avalia atitudes sobre sete doenças mentais (depressão grave, ataques de pânico ou fobias, esquizofrenia, demência, perturbação do comportamento alimentar, alcoolismo e toxi- 
codependência). As atitudes são avaliadas através de respostas a 8 itens (perigosidade, imprevisibilidade, capacidade dos médicos em falar com a pessoa, a única pessoa a culpar pela situação, sente de maneira diferente das outras pessoas, se quisesse podia melhorar a situação em que está, a pessoa pode melhorar com o tratamento e a pessoa pode recuperar por completo). Os resultados obtidos demonstraram que o facto de se conhecer alguém com esquizofrenia, demência e toxicodependência não estava relacionado com qualquer tipo de mudança de atitudes; não se verificaram mudanças de atitudes relativamente à esquizofrenia e demência, nos itens perigosidade, a culpar pela situação e se quisesse podia melhorar a situação em que está, antes e depois dos estudantes receberem formação na área da saúde mental. Em contrapartida, verificaram-se diferenças significativas antes e depois da formação em relação à toxicodependência, nos itens perigosidade e é a única pessoa a culpar pela situação, tendo havido uma diminuição de atitudes negativas (Markström et al., 2009).

\section{PARTE II}

\section{Metodologia}

\subsection{Objectivos Específicos}

1) Caracterizar as percepções de uma amostra de estudantes universitários em relação a pessoas que sofrem de diferentes doenças mentais;

2) Identificar as doenças mentais alvo de percepções mais negativas por parte de uma amostra de estudantes universitários;

3) Explorar a relação entre a área de formação dos alunos e as percepções face às doenças mentais;

4) Avaliar possíveis alterações na percepção dos estudantes entre a $1 .^{\mathrm{a}}$ e a $2 .^{\mathrm{a}}$ fase de avaliação;

5) Avaliar se os estudantes que foram expostos à campanha anti-estigma alteraram as suas percepções iniciais. 


\subsection{Questões de Investigação}

1) Quais as doenças mentais que são alvo de percepções mais negativas?

2) O curso frequentado influencia a percepção dos estudantes?

3) As percepções dos estudantes de psicologia face às doenças mentais variam entre o primeiro e o último ano de formação?

4) Os estudantes que dizem conhecer alguém com doença mental apresentam percepções face à doença mental diferentes dos alunos que dizem não conhecer?

5) Houve alteração de percepções entre a $1 .^{a}$ e a $2 .^{a}$ fase de avaliação?

6) A exposição dos estudantes à informação sobre a campanha anti-estigma contribuiu para a alteração das percepções?

\subsection{Amostra}

No início do estudo ( $1 .^{a}$ fase da avaliação), estabeleceu-se como objectivo incluir o número mínimo de 60 estudantes de cada área de formação, sendo 30 estudantes do sexo feminino e 30 do sexo masculino.

A amostra desta fase foi constituída por 285 estudantes de instituições do ensino superior público e privado, situadas no Porto. Os alunos estavam inscritos em cursos de diferentes áreas: psicologia $(n=50)$, artes ( $n=60)$, direito $(n=64)$, economia/gestão $(n=60)$ e saúde $(n=51)$, com idades compreendidas entre os 18 e os 25 anos.

A $2 .^{a}$ avaliação (pós-campanha) partiu do total dos 285 estudantes incluídos na $1 .^{a}$ fase, tendo-se eliminado 109 por falta de dados de contacto e/ou questionários incompletos. Os restantes 176 estudantes foram contactados via telefónica ou através de endereço electrónico. Destes, 50 não responderam aos emails, 57 não atenderam as chamadas telefónicas e 21 não mostraram disponibilidade para realizar o questionário. Apenas 48 estudantes efectuaram a $2 .^{a}$ avaliação. Destes, 7 da área de direito, 12 da área de artes, 21 de psicologia e 8 de economia. Dos 48 estudantes, 46 foram contactados via telefónica e 2 através do endereço electrónico. É ainda de destacar que houve um questionário feito por telefone, a um 
estudante de economia, uma vez que este não se mostrou disponível a fazê-lo presencialmente.

\subsection{Instrumento}

Com o objectivo de avaliar as percepções dos alunos sobre as doenças mentais usámos a versão traduzida do questionário utilizado na sondagem efectuada pelo Gabinete Nacional de Estatística Britânico (Office for National Statistics - ONS), no âmbito de uma campanha anti-estigma realizada pelo Royal College of Psychiatrists, para o que obtivemos a autorização dos autores.

Este questionário foi desenvolvido para informar/monitorizar o efeito da campanha anti-estigma promovida pelo Royal College of Psychiatry. Desta forma, o Comité de Acompanhamento da Campanha "Changing Minds: Every Family in the Land" solicitou ao Gabinete Nacional de Estatística uma sondagem de opinião para avaliar a percepção pública sobre pessoas com diagnóstico das sete doenças mentais que seriam alvo da campanha. Para tal, partiram de um conjunto de opiniões que as pessoas tendem a ter em relação a quem possui o diagnóstico de uma doença mental.

O inquérito engloba perguntas sobre sete doenças: depressão grave, ataques de pânico ou fobias, esquizofrenia, demência (ex. doença de Alzheimer), distúrbios alimentares (ex. anorexia nervosa, bulimia nervosa), alcoolismo e toxicodependência, começando por perguntar aos participantes se conhecem alguém com cada um dos diagnósticos.

Os participantes são, em seguida, questionados sobre oito percepções específicas que se repetem em relação a cada uma das sete doenças. Os temas abordados partiram da revisão de literatura sobre estigmatização das pessoas com doença mental, efectuada por Hayward e Bright (1997), onde os autores concluíram que existem certos temas persistentes acerca da percepção que o cidadão comum tem relativamente às pessoas com doença mental: (1) perigosas para os outros, (2) imprevisíveis, (3) difíceis de se falar com, (4) (às vezes) sentirem de forma diferente da que nós sentimos, (5) serem as únicas responsáveis pela sua condição, (6) 
que poderiam recuperar se quisessem, (7) que não melhorariam se thes fosse dado tratamento, (8) que nunca recuperarão completamente. As respostas são dadas numa escala de Likert de cinco pontos, em que os extremos representam afirmações opostas, por exemplo: perigosas para os outros - não perigosas para os outros.

Para o presente estudo foi elaborado um questionário sócio-demográfico para levantamento de alguns dados pessoais.

\subsection{Recolha de dados}

A recolha de dados foi realizada em duas fases: pré-campanha e pós campanha. A primeira fase decorreu entre Abril e Maio de 2007. Depois de obtida a autorização das respectivas direcções das faculdades e depois do treino das entrevistadoras na aplicação do instrumento, a recolha de dados foi efectuada nos espaços públicos das diferentes faculdades, durante os intervalos ou no final das aulas. A entrevista começava com uma breve explicação do estudo. Se o estudante aceitasse o pedido começava por assinar o consentimento informado e era-lhe pedido para deixar o seu contacto telefónico ou endereço electrónico para efeitos de $2 .^{a}$ avaliação. A entrevista começava com o questionário sobre percepções, terminando com a recolha de dados sócio-demográficos.

A segunda fase decorreu entre Novembro de 2008 e Janeiro de 2009. Os estudantes foram inicialmente contactados por telefone ou através de endereço electrónico e a entrevista foi marcada de acordo com a disponibilidade dos entrevistados. Os procedimentos foram idênticos aos definidos na primeira fase de avaliação, com excepção de se ter acrescentado uma pergunta, questionando os alunos sobre se estes tinham conhecimento suficiente acerca de cada uma das doenças mentais incluídas no questionário.

No estudo do Reino Unido, o questionário foi efectuado no formato de entrevista telefónica. As informações sobre amostragem e procedimentos estão descritas em Crisp, Gelder, Rix, Meltzer \& Rowlands, 2000 e Office for National Statistics (2003). 


\subsection{Tratamento de dados}

O tratamento de dados foi realizado através do software SPSS 16.0 para Windows. Foram realizadas análises descritivas com recurso a análises das frequências relativas das percepções dos estudantes face a cada doença mental e análise da média das percepções em relação ao curso, ao ano de formação, ao facto de se conhecer alguém com doença mental e à exposição à informação da campanha.

Para se avaliar se existiram diferenças entre a percepção dos estudantes e as variáveis já enumeradas recorreu-se a testes de hipóteses (testes $t$ de amostras independentes). Por último, para avaliar se houve alteração de percepções entre a $1 .^{a}$ e a $2 .^{a}$ fase de avaliação recorreu-se também a um teste de hipóteses (teste $t$ ), mas para amostras emparelhadas.

\section{Resultados}

A análise dos resultados obtidos no presente estudo obedeceu aos mesmos princípios do artigo original de Crisp, Gelder, Goddard \& Meltzer (2005), no qual é feita a análise comparativa dos resultados das duas sondagens sobre a percepção da população no Reino Unido face à doença mental, realizadas em 1998 e 2003, antes do início e no final da campanha Changing Minds.

A intenção inicial era a de apresentar os resultados relativamente a ambas as fases do estudo, porém, a amostra reduzida da 2. ${ }^{a}$ fase não permitiu concretizar o pretendido em três das questões de investigação.

Para a análise dos resultados foi criada a variável Percepção Geral (PG) para cada uma das doenças estudadas, a qual foi obtida pela soma dos valores obtidos em 5 dos 8 itens estudados. Excluíram-se os itens: "Não melhoraria mesmo com tratamento/Melhoraria se recebesse tratamento"; "Por vezes sente de maneira diferente da que nós sentimos/Por vezes sente da mesma maneira"; "Nunca irá recuperar por completo/Pode, eventualmente, recuperar". Dois dos itens omitidos referem-se ao prognóstico, porque neste caso uma resposta negativa pode reflectir conhecimento 
em vez de estigma, e um dos itens refere-se ao sentimento, dado que se reportou que alguns indivíduos acharam este item difícil de compreender (Crisp, Gelder, Goddard \& Meltzer, 2005), algo que se verificou também durante esta recolha de dados.

Para cada doença, o valor da variável "Percepção Geral” (PG) varia entre 5 e 25, sendo que 5 é a resposta mais negativa. Posteriormente foram agrupados diferentes valores em três categorias: "Negativo" (pontuação de 5 a 12), "Neutro" (pontuação de 13 a 17) e "Positivo" (pontuação de 18 a 25). Foi também criada a variável "Percepção Geral Total" (PGT) pela soma dos valores da variável "Percepção Geral" relativa às 7 doenças, a qual varia entre 35 (valor mais negativo) e 175 (valor mais positivo).

Os resultados serão apresentados em relação a cada uma das questões de investigação anteriormente enumeradas.

Quais as doenças mentais que são alvo de percepções mais negativas?

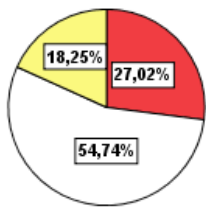

Depressão

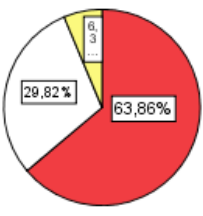

Alcoolismo

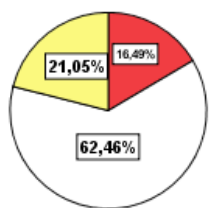

Ataques de pânicolfFobias

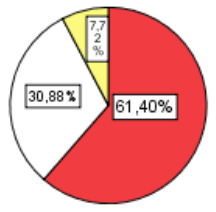

Toxicodependência

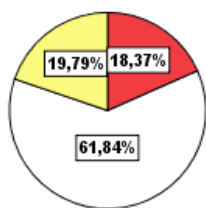

Demência

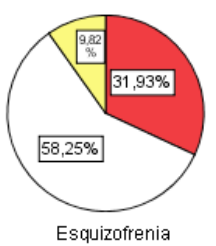

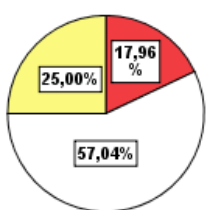

P. comportamento alimentar

$\square$ Negativa (5-12)

$\square$ Neutro (13-17)

Positivo (18-25)

Figura 1. Percentagens das percepções dos estudantes (positivas, neutras e negativas) relativamente às diferentes doenças na $1 .^{a}$ fase. 


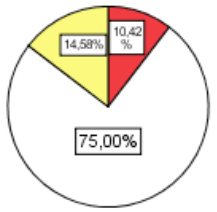

Depressẫo

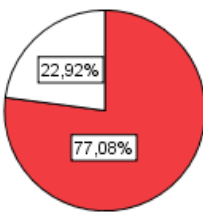

Alcoolismo

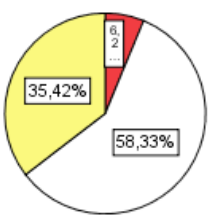

Ataques de pânicolfFobias

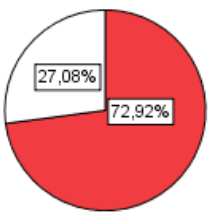

Toxicodependência

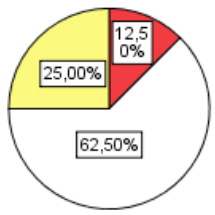

Demência

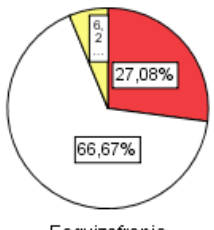

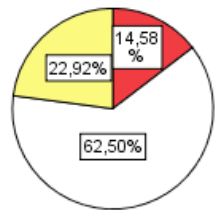

P. comportamento alimentar
Negativa (5-12)

Neutro (13-17)

Positivo (18-25)

Figura 2. Percentagens das percepções dos estudantes (positivas, neutras e negativas) relativamente às diferentes doenças na $2 .^{a}$ fase.

Conforme explícito nos gráficos acima representados, em ambas as fases do estudo as doenças que são alvo de percepções mais negativas são o alcoolismo $\left(63,86 \%\right.$ de percepções negativas para a $1 .^{\mathrm{a}}$ fase e $77,08 \%$ para a $2 .^{a}$ fase) e a toxicodependência (61,4\% de percepções negativas para a $1 .^{a}$ fase e $72,92 \%$ para a $2 .^{a}$ fase). Nas restantes doenças mentais, como pode ser observado, prevalecem percepções neutras em ambas as fases de avaliação.

\section{O curso frequentado influencia a percepção dos estudantes?}

Quadro 1. Média da PGT da $1 .^{\mathrm{a}}$ fase em relação a cada área de formação.

\begin{tabular}{|c|c|c|c|}
\hline Curso & N & Média & Desvio-Padrão \\
\hline Artes & 60 & 92,5 & 1,5 \\
\hline Direito & 64 & 100,6 & 1,3 \\
\hline Economia/Gestão & 60 & 94 & 1,5 \\
\hline Psicologia & 49 & 100,4 & 1,3 \\
\hline Saúde & 49 & 100,8 & 1,5 \\
\hline $\begin{array}{l}\text { F }=\mathbf{7 , 5 3 5} \\
\mathbf{p}=\mathbf{0 , 0 0 0}\end{array}$ & & & \\
\hline
\end{tabular}


Tendo em conta os dados da tabela acima representada, as médias da Percepção Geral Total variam entre 92,5 e 100,8. De acordo com a análise de testes post-hoc (teste Tuckey), verificou-se que as principais diferenças residem no curso de Artes e Economia/Gestão, cursos que apresentam uma média de Percepção Geral Total mais negativa (92,5 e 94, respectivamente) do que os outros cursos.

Através de uma análise ANOVA, para verificar se existem diferenças na Percepção Geral Total entre cursos, os resultados indicam que há diferenças significativas $(F=7,535 ; p=0,000)$.

Relativamente à $2 .^{a}$ fase de avaliação, dado o número reduzido de sujeitos de cada curso, não é possível proceder a análises semelhantes.

As percepções dos estudantes de psicologia face às doenças mentais variam entre o primeiro e o último ano de formação?

Quadro 2. Média da PGT no $1 .^{\circ}$ e último ano de formação, na $1 .^{a}$ fase.

\begin{tabular}{|c|c|c|c|}
\hline Ano & $\mathbf{N}$ & Média & Desvio-Padrão \\
\hline 1 & 3 & 95,7 & 6,1 \\
\hline 4 & 12 & 103,6 & 5 \\
\hline $\mathbf{t}=\mathbf{- 2 , 3 4 5}$ & & & \\
$\mathbf{p}=\mathbf{0 , 0 3 6}$ & & & \\
\hline
\end{tabular}

Somatório do $4 .^{\circ}$ e do $5 .^{\circ}$ ano

Relativamente à Percepção Geral Total dos estudantes de psicologia na $1 .^{a}$ fase de avaliação, recorreu-se a uma análise de teste de hipóteses (teste $t$ para amostras independentes). Com esta análise verificou-se que existem diferenças significativas das percepções entre os estudantes do $1 .^{\circ}$ e do último ano do curso de psicologia $(t=-2,345 ; \mathrm{p}=0,036)$. Algo que parece indicar que as percepções variam entre estes anos de formação, observando-se um valor de PGT menos negativo para os estudantes do último ano $(103,6)$.

Em relação à $2 .^{a}$ fase de avaliação, dado o número reduzido de sujeitos da amostra, não é possível proceder a análises semelhantes. 
Os estudantes que dizem conhecer alguém com doença mental apresentam percepções face à doença mental diferentes dos alunos que dizem não conhecer?

Quadro 3. Média da PG dos alunos que conhecem/não conhecem alguém com doença mental, na $1 .^{\mathrm{a}}$ fase.

\begin{tabular}{|c|c|c|c|c|c|c|c|}
\hline $\begin{array}{c}\text { Estudantes } \\
\text { que conhecem } \\
\text { alguém com } \\
\text { DM }\end{array}$ & Depressão & $\begin{array}{c}\text { Ataques } \\
\text { de Pânico }\end{array}$ & Demência & PCA & Alc. & Tox. & Esquiz. \\
\hline N & 244 & 244 & 242 & 243 & 244 & 244 & 244 \\
Sim Média & 14,5 & 15,3 & 15,2 & 15,3 & 11.2 & 11,5 & 13,7 \\
DP & 3 & 2,9 & 2,9 & 3,1 & 3,5 & 3,4 & 2,7 \\
\hline N & 41 & 41 & 41 & 41 & 41 & 41 & 41 \\
Não Média & 14,9 & 14,7 & 14,7 & 14,9 & 13,7 & 14,1 & 14,1 \\
DP & 3 & 2,3 & 3,2 & 3,3 & 3,9 & 3,4 & 3,3 \\
\hline & $\mathrm{t}=0,683$ & $\mathrm{t}=-1,308$ & $\mathrm{t}=-0,919$ & $\mathrm{t}=-0,579$ & $\mathrm{t}=3,756$ & $\mathrm{t}=4,638$ & $\mathrm{t}=0,841$ \\
& $\mathrm{p}=0,495$ & $\mathrm{p}=0,192$ & $\mathrm{p}=0,359$ & $\mathrm{p}=0,563$ & $\mathrm{p}=0,000$ & $\mathrm{P}=0,000$ & $\mathrm{p}=0,401$ \\
\hline
\end{tabular}

Relativamente às médias da Percepção Geral dos estudantes que conhecem/não conhecem pessoas com doença mental, na 1 . $^{\mathrm{a}}$ fase, a tabela mostra que não existe uma variação elevada, tendo em conta as diferentes doenças. Destacam-se os valores do Alcoolismo $(M=11,2)$ e Toxicodependência $(M=11,5)$, como valores mais negativos para os estudantes que dizem conhecer alguém com doença mental.

Com base numa análise de teste de hipóteses (teste $t$ para amostras independentes) para avaliar se existem diferenças na PG, para os estudantes que conhecem e para aqueles que não conhecem pessoas com doença mental, só se verificaram diferenças significativas no Alcoolismo $(\mathrm{t}=3,756 ; \mathrm{p}=0,000)$ e Toxicodependência $(\mathrm{t}=4,638 ; \mathrm{p}=0,000)$.

Em relação à $2 .^{a}$ fase de avaliação, dado o número reduzido de sujeitos da amostra, não é possível proceder a análises semelhantes. 
Houve alteração de percepções entre a $1 .^{a}$ e a $2 .^{a}$ fase de avaliação?

Quadro 4. Comparação da PG entre a $1 .^{\mathrm{a}}$ e a $2 .^{\mathrm{a}}$ fase de avaliação.

\begin{tabular}{|c|c|c|c|c|c|c|c|c|}
\hline Fases & Depressão & $\begin{array}{c}\text { Ataques } \\
\text { de Pânico }\end{array}$ & Demência & PCA & Alc & Tox. & Esquiz. & $\begin{array}{c}\text { Percepção } \\
\text { Geral Total }\end{array}$ \\
\hline $\begin{array}{c}1 .{ }^{\text {a }} \text { Fase } \\
\text { N }\end{array}$ & 48 & 48 & 48 & 48 & 48 & 48 & 48 & 48 \\
Média & 14,7 & 16 & 15,6 & 15,7 & 10,4 & 10,8 & 13,9 & 97,1 \\
DP & 2,6 & 2,8 & 2,9 & 3 & 3 & 3 & 2,6 & 11,4 \\
\hline $2 .{ }^{a}$ Fase & & & & & & & & 48 \\
N & 48 & 48 & 48 & 48 & 48 & 48 & 48 & 48 \\
Média & 14,2 & 16,1 & 15,8 & 15,4 & 10,3 & 10,5 & 14,4 & 97,6 \\
DP & 2,5 & 2,6 & 2,9 & 2,8 & 2,6 & 3 & 3,7 & 11,8 \\
\hline & $\mathrm{t}=-1,193$ & $\mathrm{t}=-0,044$ & $\mathrm{t}=-0,387$ & $\mathrm{t}=0,655$ & $\mathrm{t}=0,257$ & $\mathrm{t}=0,681$ & $\mathrm{t}=-0,983$ & $\mathrm{t}=-0,311$ \\
& $\mathrm{p}=0,239$ & $\mathrm{p}=0,965$ & $\mathrm{p}=0,701$ & $\mathrm{p}=0,516$ & $\mathrm{p}=0,799$ & $\mathrm{p}=0,499$ & $\mathrm{p}=0,331$ & $\mathrm{p}=0,757$ \\
\hline
\end{tabular}

Relativamente à comparação da Percepção Geral dos estudantes entre a $1 .^{\mathrm{a}}$ a e a 2. ${ }^{\mathrm{a}}$ fase de avaliação e com base numa análise de teste de hipóteses (teste $t$ para amostras emparelhadas), pode verificar-se que não existem diferenças significativas entre as duas fases $(\mathrm{t}=-0,311 ; \mathrm{p}$ $=0,757)$, o que parece indicar que não houve mudança de percepções dos estudantes.

A exposição dos estudantes à informação sobre a campanha antiestigma contribuiu para a alteração das percepções?

Quadro 5. Média da PGT dos estudantes que foram ou não expostos à informação da campanha anti-estigma.

\begin{tabular}{|l|c|c|c|}
\hline Contacto com a campanha & $\mathbf{N}$ & Média & DP \\
\hline Sim & 15 & $-0,8^{*}$ & 2 \\
\hline Não & 33 & $1,1212^{*}$ & 2,9 \\
\hline $\begin{array}{l}\mathbf{t}=\mathbf{0 , 5 2 8} \\
\mathbf{p}=\mathbf{0 , 6}\end{array}$ & & & \\
\hline
\end{tabular}

* Média da diferença entre a média da PGT dos estudantes da $1 .^{a}$ fase e a média da PGT da $2 .^{a}$ fase. 
Os dados representados na tabela acima apresentada indicam que, no que respeita à média da Percepção Geral Total dos estudantes, não existem diferenças significativas $(\mathrm{t}=0,528 ; \mathrm{p}=0,6)$ quanto à percepção dos estudantes que foram expostos à informação da campanha anti-estigma e daqueles que não foram expostos.

\section{Discussão de resultados}

\section{Percepções dos estudantes na $10^{a}$ e $2 .^{a}$ fase de avaliação}

De acordo com os resultados apresentados verifica-se uma percentagem mais elevada de percepções negativas dos estudantes universitários relativamente ao Alcoolismo e à Toxicodependência em ambas as fases de estudo. Este dado é semelhante ao encontrado no estudo de follow-up de Crisp, Gelder, Goddard \& Meltzer (2005). Quanto às restantes doenças estudadas, registou-se uma maior prevalência de percepções neutras, também nas duas fases de avaliação.

Segundo Johns (2000), a definição de estigma proposta por Goffman é também aplicada ao Alcoolismo e à Toxicodependência e qualquer pessoa familiarizada com os problemas do álcool e da toxicodependência pode sugerir possíveis razões para a existência de estereótipos negativos face a estas doenças. Por exemplo, as pessoas com estas doenças são comummente percebidas como tendo comportamentos fora do controlo e como sendo difíceis de lidar. O abuso de substâncias é visto como um comportamento auto-infligido, com elevado risco de auto-mutilação e violência em relação aos outros (Johns, 2000).

A questão da discriminação face aos indivíduos com estes tipos de doença mental complexificou-se pela marcada ambivalência da sociedade em relação ao consumo de droga e álcool. Por exemplo, no século XIX, o consumo de opiáceos era considerado refinado. A cocaína foi inicialmente descrita por Freud como uma substância que não causava dependência (Johns, 2000). 
Ainda de acordo com Johns (2000), as percepções gerais das pessoas com alcoolismo e toxicodependência são complexas: um jovem que revele um comportamento ameaçador e perturbado e que the tenha sido diagnosticado esquizofrenia, é visto como alguém que não pode ser culpado pela sua situação e que merece tratamento. Por outro lado, o comportamento de um jovem que está ligado ao consumo de droga ou álcool é frequentemente visto como culpado pela sua situação e, por isso, não merece o acesso a tratamento médico adequado.

Considerar o consumo excessivo de álcool ou droga como uma escolha, é talvez a causa determinante de estigma, ou seja, se o indivíduo escolhe ser alcoólico o seu comportamento ofensivo é comum ser alvo de punição por parte da sociedade, tal como qualquer outro comportamento ofensivo (Johns, 2000).

Com efeito, a percentagem dos estudantes neste estudo que atribuíram culpa aos indivíduos com alcoolismo e toxicodependência rondou os 50\%, algo que podemos considerar enquadrar no princípio acima referido.

Em Portugal, a questão da toxicodependência é vivida com algum receio. Cerca de $80 \%$ dos estudantes desta amostra revelaram percepções negativas ao atribuir perigosidade a estas doenças. Muito frequentemente os meios de comunicação social divulgam acontecimentos violentos relacionados com indivíduos com problemas de abuso de substâncias ilícitas (homicídios, roubos), transmitindo uma imagem destes indivíduos como perigosos. Numa conferência internacional de debate sobre a toxicodependência em Portugal, em 2007, divulgou-se que 53\% dos crimes estavam associados à toxicodependência.

Para além disto, mais recentemente, a figura dos "arrumadores de carros" que apareceu em muitas cidades, está muitas vezes associada à figura de uma pessoa com toxicodependência. Muitos deles são temidos pela maioria das pessoas que se sente obrigada a dar dinheiro para o seu carro se manter intacto. Obviamente que esta situação, aparentemente coerciva, desenvolve uma visão ainda mais negativa da população face à toxicodependência. 


\section{As percepções dos estudantes nas diferentes áreas de formação estudadas}

As únicas diferenças de percepções observaram-se nos cursos de Artes e Economia/Gestão que apresentam percepções mais negativas do que os outros cursos.

Apesar de a literatura demonstrar a existência de percepções estigmatizantes em pessoas com diferentes formações, também é verdade que um dos elementos que está na base de estigma diz respeito à "ignorância" sobre esta problemática (Thornicoft, Rose, Kassam \& Sartorius, 2007). Neste sentido, é de esperar que os estudantes com formação na área da saúde, por terem contacto com esta problemática durante os seus cursos, revelem percepções menos negativas face à doença mental.

\section{A variação das percepções dos estudantes de psicologia entre o $1 .^{\circ}$ e o último ano de formação}

Verificaram-se diferenças significativas nas percepções dos estudantes de psicologia entre o $1 .^{\circ}$ e o último ano de formação. Esta diferença era já esperada, na medida em que estes estudantes vão tendo um contacto gradual com esta problemática ao longo do curso. Ainda que o conhecimento não seja o único elemento que pode desenvolver percepções e atitudes estigmatizantes (Thornicoft, Rose, Kassam \& Sartorius, 2007), espera-se que, à medida que o conhecimento aumente, as percepções negativas diminuam. Tendo em conta o estudo de Holmes, Corrigan, Williams, Canar \& Kubiak (1999), que comparou estudantes adultos de um curso de psicopatologia com uma turma de estudantes de psicologia introdutória, verificou-se que todos os estudantes melhoraram as suas atitudes face à doença mental.

De acordo com Feldmann (2005), e em relação aos alunos de medicina, é provável que a maioria dos estudantes ingresse na faculdade de medicina com um conhecimento limitado sobre doenças psiquiátricas. Alguns estudos existentes demonstram que a formação académica influencia favoravelmente as mudanças de atitudes dos estudantes face à doença 
mental (Singh, Baxter, Standen \& Duncan, 1998, cit. in Mino, Yasuda, Kanazawa \& Inoue, 2000)

O estudo de Mino, Yasuda, Kanazawa \& Inoue, (2000) avaliou os efeitos de 5 anos de formação académica em medicina, os conteúdos programáticos das disciplinas e a experiência clínica dos alunos. Os resultados sugerem que a formação académica nesta área influencia a aceitação das pessoas com doença mental, as atitudes quanto aos serviços psiquiátricos e as atitudes acerca da doença mental em geral.

De acordo com os mesmos autores, a formação académica em medicina é fundamental para o desenvolvimento de atitudes positivas, não só dos estudantes, mas consequentemente do público em geral, podendo contribuir para uma melhoria nos serviços de saúde mental.

No estudo de Markström et al., (2009) que procurou avaliar a mudança de atitudes dos estudantes universitários na área da saúde, como já foi referenciado, observaram-se diferenças significativas de atitudes antes e depois dos estudantes receberem formação, tendo estes diminuído as atitudes negativas em relação à toxicodependência.

Com efeito, uma das estratégias usadas para diminuir o estigma público é a educação (Corrigan \& Penn, 1999; Corrigan \& Watson, 2002). De acordo com Corrigan e Watson (2002), os programas educativos acerca da doença mental são eficazes para diversos grupos, incluindo os estudantes universitários. Isto porque ao fornecer informação acerca da doença mental podem contribuir para promover atitudes mais positivas (Stuart, 2003).

\section{A influência de conhecer/não conhecer pessoas com doença mental na percepção dos estudantes}

Os dados analisados revelam que apenas existem diferenças entre conhecer e não conhecer pessoas com doença mental em relação ao alcoolismo e à toxicodependência. Porém, o que não seria esperado era que os estudantes que conhecem indivíduos com este tipo de doenças demonstrassem percepções mais negativas, do que aqueles que não conhecem, conforme aconteceu. 
De acordo com Crisp, Gelder, Rix, Meltzer \& Rowlands (2000), o efeito do contacto com indivíduos com doença mental depende da natureza do contacto e da natureza da doença mental. Tal como estes autores defendem no seu estudo, também neste estudo é provável que as percepções acerca do comportamento perigoso dos indivíduos com as doenças supracitadas tenham sido mais influenciadas por algum acontecimento dramático recente difundido pelos meios de comunicação social e que estivesse ligado à toxicodependência e alcoolismo. Conforme já referido, temos acesso frequente a notícias relacionadas com crimes violentos associados à toxicodependência. Este facto pode explicar, assim, a ausência de influência do contacto com alguém com doença mental nas percepções. Neste sentido, as campanhas de combate ao estigma deverão ter em atenção a informação que é difundida pelos meios de comunicação social.

No estudo de follow-up da campanha inglesa Changing Minds também não se registaram diferenças significativas entre conhecer e não conhecer pessoas com doença mental, verificando-se que, aparentemente, conhecer pessoalmente alguém com doença mental nem sempre modifica as opiniões negativas que os indivíduos têm (Crisp, Gelder, Goddard \& Meltzer, 2005). Contudo, há evidência de que o contacto informado com pessoas com doença mental grave pode levar a um aumento de conhecimento e de empatia na população em geral (Wolff, Pathare \& Craig, 1996, cit. in Crisp, Gelder, Goddard \& Meltzer, 2005).

Comparação das percepções dos estudantes entre a 1 . $^{\mathrm{a}}$ e a $2 .^{\mathrm{a}}$ fase de avaliação

Através dos resultados obtidos verificou-se que não existiram diferenças de percepções dos estudantes entre a $1 .^{\mathrm{a}}$ e a $2 .^{\mathrm{a}}$ fase de avaliação. No estudo de follow-up da campanha Changing Minds, pelo contrário, foram observadas diferenças estatisticamente significativas em 27, dos 56 itens analisados, tendo havido, à excepção das opiniões sobre o tratamento, diminuição nas percentagens das opiniões negativas (Crisp, Gelder, Goddard \& Meltzer, 2005). 
Um dos objectivos das campanhas anti-estigma/pró-saúde mental é o de contribuir para a substituição dos estereótipos relacionados com a doença mental que estão ligados à discriminação e ao isolamento das pessoas que sofrem deste tipo de doença, no intuito de se melhorarem atitudes. Numa primeira análise, uma vez que não se registaram diferenças significativas de percepções na amostra em estudo entre a $1 .^{\mathrm{a}}$ e a $2 .^{\mathrm{a}}$ fase, pode parecer que a campanha não surtiu o efeito desejado nesta amostra de alunos. No entanto, existem vários aspectos que merecem ser considerados uma vez que as opiniões estão sujeitas a muitas outras influências. Para além disto, em primeiro lugar, o número reduzido da amostra que foi comparada (48 estudantes) é insuficiente para se generalizarem conclusões à população dos estudantes universitários.

Em segundo lugar, a mudança de percepções envolve um processo complexo influenciado por diferentes factores. O movimento UPA tinha como objectivo informar a população sobre os temas da doença mental e do estigma, não tendo havido nenhuma campanha prévia mais específica no país. Desta forma, para haver mudança de percepções teria sido necessário que os estudantes tivessem procurado por eles próprios esclarecer algumas questões sobre a temática em estudo, o que não nos foi possível avaliar.

\section{A influência da exposição à campanha anti-estigma nas percepções dos estudantes}

Quando analisadas as percepções dos estudantes que foram expostos e dos que não foram expostos à informação da campanha anti-estigma, também não encontramos diferenças significativas. Como já foi referido anteriormente, as percepções podem ser influenciadas por muitos factores para além das campanhas anti-estigma. Segundo McGuire (1985, cit. in Wallach, 2004), as atitudes desenvolvem-se na infância e, mais tarde a sociedade, principalmente os meios de comunicação social, continuam a modificá-las. 
A televisão, a rádio e os jornais desempenham um importante papel na percepção do público em geral em relação à doença mental (Salter \& Byrne, 2000). As imagens que são apresentadas sobre indivíduos com este tipo de doença formam as atitudes do público influenciando os seus comportamentos (Jorge-Monteiro \& Madeira, 2007).

Segundo Crisp, Gelder, Goddard \& Meltzer (2005), os meios de comunicação social focam ainda, muito frequentemente, a atenção do público para as características mais negativas da doença mental, contribuindo para aumentar percepções negativas. Eles caracterizam as pessoas com doença mental como estando desempregadas e como sendo perigosas e imprevisíveis (Malla \& Shaw, 1987 cit. in Stuart 2003).

A experiência de contacto com algum amigo ou familiar com este tipo de doença (Thornicoft, 2006 cit. in Markström et al., 2009) e a educação, como já referido, são também considerados importantes meios possíveis de mudança de atitudes.

A estratégia da educação por si só não promove mudanças eficazes no comportamento das pessoas, já que os estereótipos podem tornar-se muito resistentes a nova informação (Jorge-Monteiro, 2006). Deste modo, a experiência de contacto directo assume uma grande importância e esta experiência pode ser variada: uma visita a um hospital psiquiátrico, que pode incluir uma visita guiada onde os estudantes possam ver os pacientes e ter acesso a alguma informação acerca da forma como eles vivem e como são tratados (Wallach, 2004). Um outro tipo de experiência pode acontecer quando os estudantes fazem voluntariado numa instituição deste tipo. Por fim, salienta-se o tipo de experiência de contacto pessoal no qual há interacção com a pessoa com doença mental fora da instituição (Wallach, 2004).

\section{Limitações do estudo}

Este estudo foi desenvolvido no âmbito de uma campanha anti-estigma/ pró-saúde mental, pioneira em Portugal, para compreender as percepções 
dos estudantes universitários face à doença mental antes e depois da implementação desta mesma campanha.

As principais limitações deste estudo estão ligadas a questões metodológicas. Em primeiro lugar uma das dificuldades presentes foi a de manter o número da amostra inicial (285 estudantes) uma vez que da $1 .^{a}$ para a $2 .^{a}$ fase de avaliação houve um decréscimo em $84 \%$ da amostra (48 estudantes).

Em segundo lugar, ao longo da aplicação do questionário três dos oito itens existentes suscitaram algumas dúvidas, algo que levou a que fossem excluídos na análise estatística. Os itens em causa são: "Não melhoraria mesmo com tratamento/Melhoraria se recebesse tratamento"; "Nunca irá recuperar por completo/Pode, eventualmente, recuperar"; "Por vezes sente de maneira diferente da que nós sentimos/Por vezes sente da mesma maneira”. Os dois primeiros itens referem-se ao prognóstico e, neste caso, uma resposta negativa pode significar conhecimento em vez de estigma, por parte dos inquiridos. Por exemplo, na Demência mais especificamente na Demência do Tipo Alzheimer, que se caracteriza por um declínio cognitivo progressivo (American Psychiatric Association, 2002), dar uma resposta negativa (1 ou 2) não reflecte uma opinião estigmatizante.

Em relação ao último item foi difícil de compreender exactamente o que se entende por "sentir de maneira diferente" ou "sentir da mesma maneira", por parte da maioria dos estudantes e, por isso, estes apresentaram dificuldades em responder. Estas são questões com as quais nos deparámos num estudo piloto e numa primeira utilização de um questionário, apesar de se compreender a utilidade dos resultados para fundamentar e para introduzir as correcções necessárias à produção de dados mais fidedignos e relevantes.

Em terceiro lugar, as comparações entre as duas fases de avaliação foram realizadas dentro dos limites estatísticos provocados pelo "drop out" da amostra na 2 . $^{\text {a }}$ fase. O número reduzido da amostra impossibilitou, assim, a comparação entre a $1 .^{a}$ e $2 .^{a}$ fase em relação a algumas das questões de investigação. 


\section{Conclusão}

Através deste estudo que teve como objectivo, avaliar as percepções dos estudantes universitários para compreender possíveis necessidades de intervenção, foi possível concluir que nesta amostra existe uma marcada prevalência de percepções negativas relativamente ao alcoolismo e toxicodependência e de percepções neutras face às restantes doenças. Estes resultados parecem revelar a presença de estigma entre os estudantes e talvez a ausência de informação e de conhecimento em relação à doença mental, dadas as elevadas percentagens de percepções neutras que se observaram nas restantes doenças.

Neste sentido, em primeiro lugar surge a necessidade de se implementar no país um maior número de campanhas de redução do estigma que desenvolvam intervenções/programas educativos sobre a doença mental e o seu impacto, com o propósito de diminuir a "ignorância" sobre esta problemática que, como já foi mencionado, é um dos elementos que desenvolve estigma. Para isso pode recorrer-se à criação de alguns materiais que permitam sensibilizar e informar as pessoas, levando-as a compreender melhor a doença mental, tais como a criação de panfletos, por exemplo, com informação sobre cada doença, brochuras para serem distribuídas nas escolas, para que desde cedo os jovens possam desenvolver percepções mais positivas sobre esta temática e vídeos. Esters et al. (1998, cit. in Wallach, 2004) constatou que mostrar um vídeo a adolescentes e fornecer-lhes informação acerca da etiologia, da sintomatologia, do diagnóstico e do prognóstico da doença mental, bem como sobre a realidade do estigma associado a este tipo de doença, era importante para melhorar significativamente as atitudes dos jovens.

Estas campanhas devem dar especial atenção aos estudantes universitários, principalmente àqueles que no futuro terão um contacto mais directo com esta problemática (psicólogos, médicos, psiquiatras, terapeutas, enfermeiros, entre outros). Em segundo lugar talvez faça sentido, nestes cursos, a criação de programas curriculares específicos voltados para a área da saúde mental e para alertar os alunos para a importância desta 
temática fundamental na área da saúde pública. Promover a oportunidade de contacto directo destes estudantes com pessoas com doença mental, por exemplo através de visitas a um hospital psiquiátrico ou, idealmente, através do contacto directo com pessoas com este tipo de doença que estejam integradas na comunidade também se revela bastante eficaz na redução da discriminação. De facto, a estratégia de contacto assume uma importância fundamental. De acordo com Read \& Harré (2001), o contacto directo com pessoas com doença mental revelou estar ligado a atitudes mais positivas por parte da população. Nos estudos realizados a estratégia de contacto directo demonstrou melhores resultados na mudança de atitudes do público.

A combinação destes destas duas estratégias - contacto directo e educação - torna-se assim particularmente eficaz na redução de estigma (Corrigan \& Penn, 1999) e é importante avaliar se estes métodos são eficazes na redução de atitudes estigmatizantes se forem incluídos nos programas curriculares ou na estrutura da prática clínica dos estudantes da área da saúde (Sadow \& Ryder, 2008).

No intuito de fornecer informação sobre a natureza, as causas e o tratamento da doença mental, os programas de intervenção devem focalizar-se também na difusão de visões mais positivas, nos meios de comunicação social, com critérios de selecção das políticas de publicação bem definidos. Tendo em conta o estudo de Jorge-Monteiro \& Madeira (2007), os resultados revelaram que a maioria dos participantes afirmam que a política de publicação em relação às questões de saúde mental não se baseia em critérios diferenciados de publicação nesta temática e que os critérios utilizados prendem-se apenas com a actualidade da notícia e se esta apresenta interesse público.

Sugere-se igualmente que se prossiga o trabalho de investigação associado aos estudantes universitários, podendo explorar-se outros factores, por exemplo, factores individuais como o género e recorrendo-se a amostras mais alargadas no intuito dos resultados poderem vir a ser generalizados a esta população. 


\section{Referências bibliográficas}

AJZEN, I. \& FISHBEIN, M. (1977). Attitude-behavior relations: a theoretical analysis and review of empirical research. Psychological Bulletin, 5, 888-918.

AMERICAN PSYCHIATRIC ASSOCIATION (2002). DSM-IV-TR - Manual de Diagnóstico e Estatística das Perturbações Mentais. $4 .^{a}$ Edição. Lisboa: CLIMEPSI Editores.

CHUnG, K., CHEN, E. \& LIU, C. (2001). University students' attitudes towards mental patients and psychitric treatment. International Journal of Social Psychiatry, 47 (2), 63-72.

CORRIgAN, P. \& PENN, D. (1999). Lessons From Social Psychology on Discrediting Psychiatric Stigma. American Psychologist, 54, 765-776.

CORRIGAN, P. \& WATSON, A. (2002). Understanding the impact of stigma on people with mental illness. World Psychiatry, 1, 16-20.

CRISP, A., GELDER, M., GODDARD, E. \& MELTZER, H. (2005). Stigmatization of people with mental illness: a follow-up study within the Changing Minds campaign of the Royal College of Psychiatrists. World Psychiatry, 4, 106-113.

CrisP, A., Gelder, M., RIX, S., Meltzer, H. \& ROwlands, O. (2000). Stigmatisation of people with mental illnesses. The British Journal of Psychiatry, 177, 4-7.

ECKER, D. (1988). University students' attitudes toward mental patients in a developing country. Social Psychiatry and Psychiatric Epidemiology, 23, 264-266.

FELDMANN, T. (2005). Medical students' attitudes toward psychiatry and mental disorders. Academic Psychiatry, 4, 354-356.

HAYWARD, P. \& BRIGHT, J. (1997). Stigma and mental illness: a review and critique. Journal of Mental Health, 6, 345-354.

Holmes, E., Corrigan, P., Williams, P., CANAR, J. \& KubiaK, M. (1999). Changing attitudes about schizophrenia. Schizophrenia Bulletin, 25 (3), 447-456.

JAVED, Z., NAEEM, F., Kingdon, D., IRFAN, M., IRZHAR, N. \& AYUb, M. (2006). Attitudes of the university students and teachers toward mentally ill, in Lahore, Pakistan. Journal of Ayub Medical College Abbottabad, 18 (3), 55-58.

JOHnS, A. (2000). Drug and Alcohol Addiction. In A. Crisp (Ed.), Every family in the land: Understanding prejudice and discrimination against people with mental illness (pp.175180). London: RSM Press.

JORGE-Monteiro, F. (2006). Prevenção do Estigma Social da Doença Mental. Saúde Mental e Reabilitação. Folhetos SNR, 56, 16-23.

JORGE-MONTEIRO, F. \& MADEIRA, T. (2007). Considerações sobre doença mental e comunicação social. Análise Psicológica, 1, 97-109.

LAuber, C., Anthony, M., Ajdacic-Gross, V. \& Rossler, W. (2004). What about psychiatrists' attitude to mentally ill people? European Psychiatry, 19, 423-427.

LEFLEY, H. (1992). The stigmatized family. In P. Fink \& A. Tasman (Eds.), Stigma and mental illness (pp.127-138). Washington, DC: American Psychiatric Press.

LIMA, L. (2004). Atitudes: Estrutura e mudança. In J. Vala \& B. Monteiro (Eds.), Psicologia Social. 6. ${ }^{a}$ Edição (pp.187-225). Lisboa: Fundação Calouste Gulbenkian.

MARKSTRÖM, U., Bejerholm, U., BJÖRKMAN, T., BRUNT, D., HANSSON, L., LeufSTAdius, C., SANDlund, M., SVEnsson, B., Östman, M. \& EKLund, M. (2009). Attitudes towards mental illness among health care students at Swedish universities - A follow-up study after completed clinical placement. Nurse Education Today, 1, 1-6. 
MinO, Y., YASUDA, N., KANAZAWA, S. \& INOUE, S. (2000). Effects of medical education on attitudes towards mental illness among medical students. Acta Medica Okayama, 3, 127-132.

OFFICE FOR NATIONAL STATISTICS (2003). Statistical omnibus survey package: technical report. London: Office for National Statistics.

RABKIN, J., MUHLIN, G. \& COHEN, P. (1984). What neighbors think: community attitudes toward local psychiatric facilities. Community Mental Health Journal, 20 (4), 304-312.

READ, J. \& HARRÉ, N. (2001). The role of biological and genetic causal beliefs in the stigmatisation of 'mental patients'. Journal of Mental Health, 10 (2), 223-235.

ROMAN, P. \& FLOYD, H. (1981). Social acceptance of psychiatric illness and psychiatric treatment. Social Psychiatry, 16, 21-29.

RÜSCH, N., ANGERMEYER, M. \& CORRIGAN, P. (2005). Mental illness stigma: concepts, consequences, and initiatives to reduce stigma. European Psychiatry, 20, 529-539.

SADOW, D. \& RYDER, M. (2008). Reducing stigmatizing attitudes held by future health professionals: the person is the message. Psychological Services, 4, 362-372.

SAlTER, M. \& BYRne, P. (2000). The stigma of mental illness: how you can use media to reduce it. Psychiatric Bulletin, 24, 281-283.

SEGAL, S., BAUMOHL, J. \& MOYLES E. (1980). Neighborhood types and community reaction to the mentally ill: A paradox of intensity. Journal of Health and Social Behavior, 21, 345-359.

SERVAIS, L. \& SAUNDERS, S. (2007). 'Clinical psychologists' perceptions of persons with mental illness. Professional Psychology: Research and Practice, 2, 214-219.

STUART, H. (2003). Stigma and Stigma Reduction: Lessons Learned. Santé Mentale au Québec, $28,54-72$.

Thompson, A., Stuart, H., Bland, R., Arboleda-Florez, J., WARner, R. \& DiCKson, R. (2002). Attitudes about schizophrenia from the pilot site of the WPA worldwide campaign against the stigma of schizophrenia. Social Psychiatry and Psychiatric Epidemiology., 37, 475-482.

ThOrnicoft, G., ROSE, D., KASSAM, A. \& SARTORIUS, N. (2007). Stigma: ignorance, prejudice or discrimination? British Journal of Psychiatry, 190, 192-193.

WAllach, S. (2004). Changes in Attitudes Towards Mental Illness Following Exposure. Community Mental Health Journal, 40, 235-248.

WORLD HEALTH ORGANIZATION (2001). The World Health Organization Report 2001-Mental Health: new understanding and hope. Geneva: World Health Organization. 